\title{
pTcGW plasmid vectors 1.1 version: a versatile tool for Trypanosoma cruzi gene characterisation
}

\author{
Fernanda G Kugeratski, Michel Batista, Alexandre Haruo Inoue, \\ Bruno Dias Ramos, Marco Aurelio Krieger, Fabricio K Marchini/+ \\ Fundação Oswaldo Cruz, Instituto Carlos Chagas, Laboratório de Genômica Funcional, Curitiba, PR, Brasil
}

\begin{abstract}
The functional characterisation of thousands of Trypanosoma cruzi genes remains a challenge. Reverse genetics approaches compatible with high-throughput cloning strategies can provide the tool needed to tackle this challenge. We previously published the $p \mathrm{Tc} G W$ platform, composed by plasmid vectors carrying different options of $N$-terminal fusion tags based on Gateway ${ }^{\circledR}$ technology. Here, we present an improved 1.1 version of $p \mathrm{Tc} G W$ vectors, which is characterised by a fully flexible structure allowing an easy customisation of each element of the vectors in a single cloning step. Additionally, both $N$ and C-terminal fusions are available with new tag options for protein complexes purification. Three of the newly created vectors were successfully used to determine the cellular localisation of four T. cruzi proteins. The 1.1 version of $p \mathrm{Tc} G W$ platform can be used in a variety of assays, such as protein overexpression, identification of protein-protein interaction and protein localisation. This powerful and versatile tool allows adding valuable functional information to T. cruzi genes and is freely available for scientific community.
\end{abstract}

Key words: Trypanosoma cruzi - plasmid vectors - pTcGW platform - reverse genetics - cloning - gene characterisation

The sequencing of Trypanosoma cruzi genome revealed a high percentage of genes assigned with unknown function or with a role attributed only based on sequence similarity (El-Sayed et al. 2005, Weatherly et al. 2009). In order to improve this scenario and to add functional information to $T$. cruzi genes, our group previously constructed the $\mathrm{p} T c \mathrm{GW}$ platform, which is composed by plasmid vectors containing several $\mathrm{N}$-terminal fusion tags (Batista et al. 2010). In the current work we improved the previous 1.0 version of the $\mathrm{p} T c \mathrm{GW}$ platform and created the $\mathrm{p} T c \mathrm{GW}$ 1.1 version by implementing three changes.

First, in order to make our platform fully flexible, we aimed that each restriction site was present only once in the vectors. For this, we replaced the $\mathrm{XhoI}$ restriction site 5 '-flanking the resistance marker by side by side $A s c \mathrm{I}$ and AgeI sites through two cloning steps. The obtained plasmid (pTc6HisP-NH 1.1) containing N-terminal hexahistidine fusion tag comprises the backbone $\mathrm{NH}$-vector of pTc GW platform 1.1 version (modification steps described in Supplementary Fig. 1). Second, to create vectors containing C-terminal fusion tags, the cassette comprising the $18 \mathrm{~S}$ ribosomal promoter (RP), the first intergenic region (IR) (TcUIR), start codon and the N-terminal 6His tag were excised from $\mathrm{p} T c 6 \mathrm{HisP}-\mathrm{NH} 1.1$ and replaced by a new cassette containing only the RP and $T c$ UIR. Next, we

doi: 10.1590/0074-02760150074

Financial support: CNPq, IBMP

MAK is research fellow from CNPq. FGK and MB contributed

equally to this work.

+Corresponding author:marchinifk@fiocruz.br

Received 22 February 2015

Accepted 23 June 2015 replaced the phleomycin resistance marker by neomycin and added a green fluorescent protein (GFP) fusion tag creating the $\mathrm{p} T c \mathrm{GFPN}-\mathrm{CO}$ 1.1, backbone for the $\mathrm{CO}$-vectors (Supplementary Fig. 2). Third, for protein complex purification, we added either protein C (ProtC) or ProtCTEV-ProtA (PTP) tags [PTP amplified from Schimanski et al. (2005)] in both $\mathrm{N}$ and C-terminal vectors.

Finally, in order to broaden the applicability of these tools towards different assays we replaced the tag and/or resistance marker through single cloning steps, generating five different amino 1.1 and four carboxy 1.1 plasmids (Fig. 1). Our destination vectors allow performing a range of functional assays to characterise T. cruzi genes, such as protein overexpression, identification of interactors and protein localisation. The sequences of the plasmids are available on GenBank (accessions described on Supplementary data 3). The sequences of primers here employed and all material and methods can be found in Supplementary data 4. We also provide technical details for users in the $\mathrm{p} T c \mathrm{GW}$ Platform Guideline. Briefly, the guide contains an explanation of how the Gateway ${ }^{\circledR}$ cloning system works, instructions for primers design, recombination reactions, plasmids propagation, T. cruzi transfection and selection.

The basic structure of the platform characterised by the presence of a strong RP (18S), IR from the ubiquitin locus (TcUIR) and the Gateway ${ }^{\circledR}$ cloning system are maintained from version 1.0. The RP, recognised by RNA polymerase I, ensures an efficient transcription of genes under its control and had already been successfully used in T. cruzi vectors (Martinez-Calvillo at al. 1997, Vazquez \& Levin 1999). IRs provide signals to drive the processing of pre-mRNAs transcribed in the long polycistronic units. In order to be functional T. cruzi mRNAs receive a 39-nucleotide splice-leader in the 5' region and a PolyA tail in the 3' region (Liang at al. 2003). Given the pivotal role of IRs, the functional elements of our 
Amino vectors
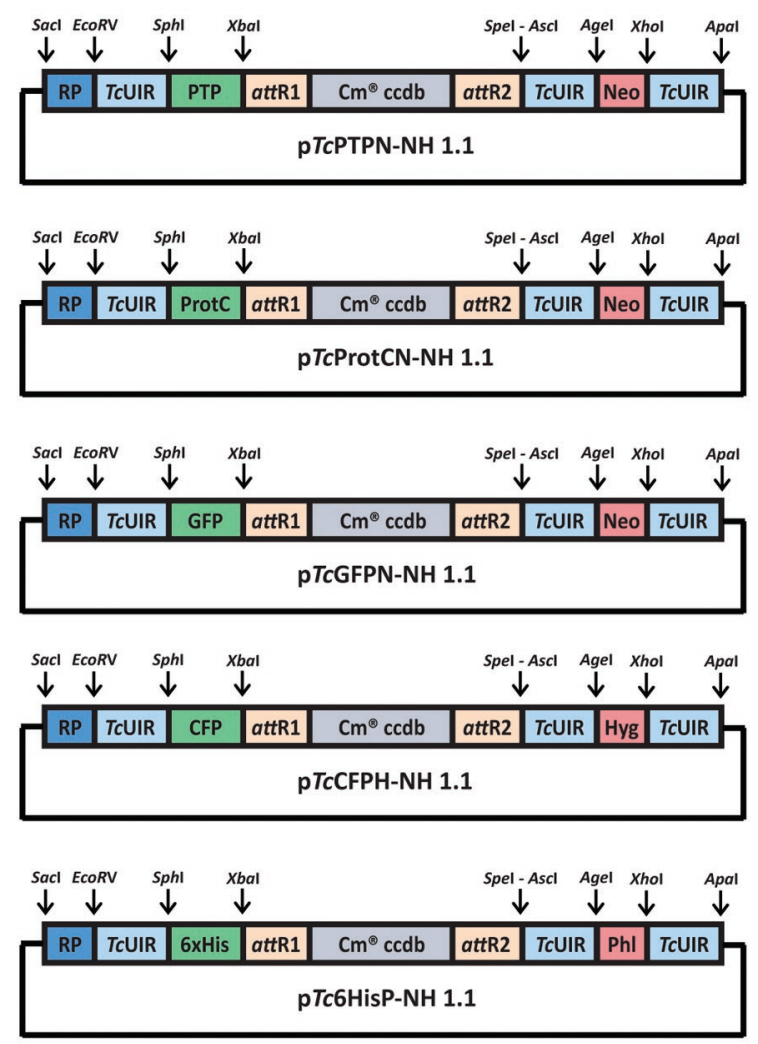

\section{Carboxy vectors}
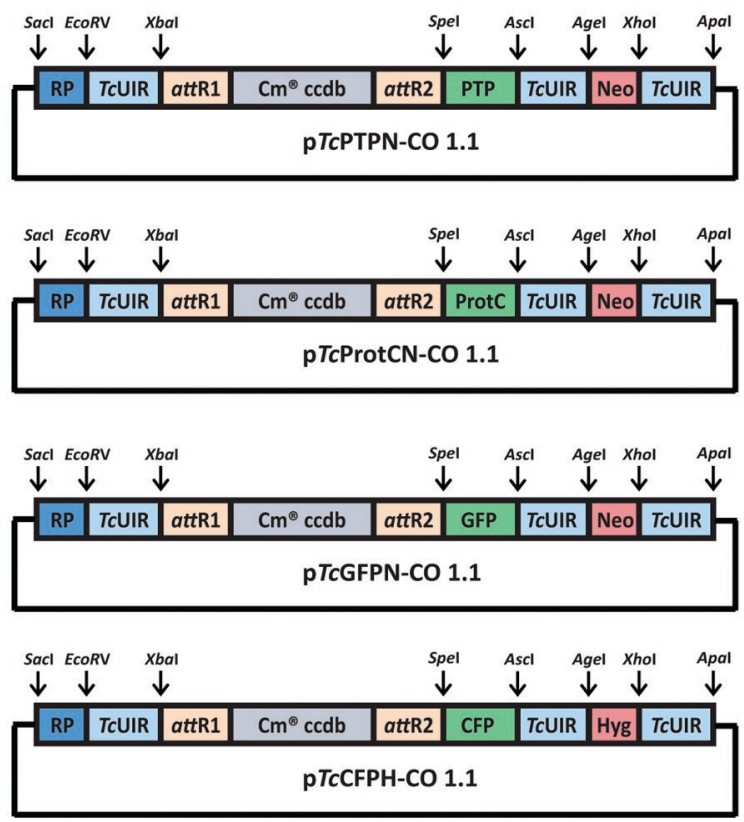

Fig. 1: available pTcGW 1.1 vectors with amino and carboxy-terminal fusion tags. Arrows indicate the restrictions sites flanking each element of the vectors. att: attachment; attR1 and attR2: attachment sites required for Gateway ${ }^{\circledR}$ recombination; $c c d \mathrm{~B}$ : gene for negative selection; $\mathrm{Cm}^{\circledR}$ : chloramphenicol selectable marker; $\mathrm{CO}$ : carboxy-terminal fusion; H: hygromycin; N: neomycin; NH: amino-terminal fusion; P: phleomycin; p: plasmid; RP: ribosomal promoter; Tag: the fusion tag used, such as green fluorescent protein (GFP), cyan fluorescent protein (CFP), ProtCTEV-ProtA (PTP), protein C (ProtC) or hexahistidine (6xHis); Tc: Trypanosoma cruzi; TcUIR: T. cruzi ubiquitin intergenic region.

vectors are flanked by $T c$ UIR, which had already been used in a T. cruzi vector (Wen at al. 2001) and is present three times in our system. Additionally, our destination vectors contain a Gateway ${ }^{\circledR}$ cassette composed by the attachment (att) sites attR1 and attR2 required for recombination, the chloramphenicol selectable marker and $c c d \mathrm{~B}$ gene for negative selection.

Here, to demonstrate that the modifications in the platform preserved its functionality, the plasmids $\mathrm{pTCPTPN}$ NH 1.1, pTcPTPN-CO 1.1 and pTcGFPN-CO 1.1 were used for protein overexpression and localisation assays.

The $117 \mathrm{kDa}$ exportin Crm1 (TcCLB.511725.150) was cloned into the N-terminal PTP plasmid ( $\mathrm{p} T c \mathrm{P}$ TPN-NH 1.1) whereas the $67 \mathrm{kDa}$ mRNA export factor Mex67 (TcCLB.506127.20) and the $95 \mathrm{kDa}$ nucleoporin Nup95 (TcCLB.510181.50) were inserted in the C-terminal PTP plasmid (pTcPTPN-CO 1.1). Fig. 2A shows that the recombinant proteins were successfully expressed with the expected molecular weight. Furthermore, T. cruzi Mex67 and Nup95 demonstrated nuclear localisation, same as described for the Trypanosoma brucei orthologue proteins (Kramer et al. 2012, Dostalova et al. 2013), whereas the exportin Crm1, which shuttles between nucleus and cytoplasm, was observed to localise in both compartments (Fig. 2B).
To exemplify the usefulnes of $\mathrm{p} T c \mathrm{GW}$ vectors to characterise hypothetical proteins we used the plasmid pTcGFPN-CO 1.1 to clone the $58.1 \mathrm{kDa}$ conserved hypothetical protein TcCLB.506825.40. The polyclonal serum raised using the recombinant protein recognised both the endogenous and the GFP-tagged protein in epimastigotes and metacyclic trypomastigotes forms of $T$. cruzi (Fig. 2C). Additionally, the intracellular localisation of the GFP-tagged TcCLB.506825.40 could be determined, which is concentrated in the posterior region of $D m 28 \mathrm{c}$ epimastigotes (Fig. 2D).

A striking feature of $\mathrm{p} T c \mathrm{GW} 1.1$ version refers to the versatility of the system. All elements of the 1.1 vectors are flanked by exclusive restriction sites allowing an easy exchange of each element of the vector in a single cloning step, such as promoter, fusion tags, resistance markers and IRs. This feature empowers the system through the range of opened options. For example, one can easily adapt the vectors for use in other trypanosomatid species by simply changing IRs and promoter if required. Furthermore, by selecting different IRs containing developmentally regulated 3'UTR signals, the expression can be controlled in a life-cycle stage dependent manner (Coughlin et al. 2000, Clayton \& Shapira 2007). Additionally, colocalisation studies can be performed by combining different tags with resistance markers. 

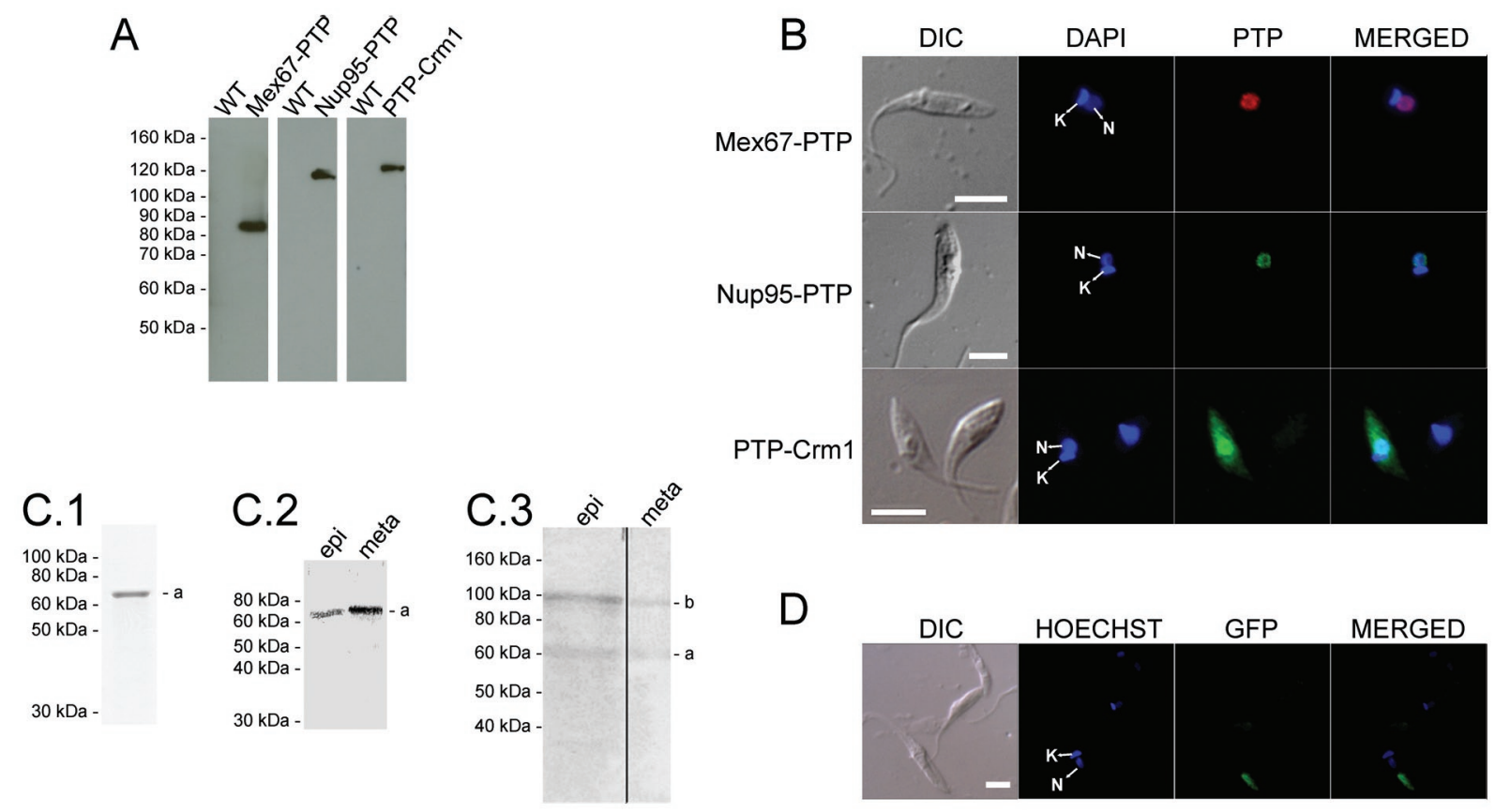

Fig. 2: cellular localisation and overexpression of tagged proteins in Trypanosoma cruzi. A: western blot (WB) using total extract of epimastigotes wild-type (WT) and expressing ProtC-TEV-ProtA (PTP)-tagged proteins; B: detection of mRNA export factor Mex67 and nucleoporin Nup95 tagged at C-terminal extremes and nuclear export factor $\mathrm{Crm} 1$ tagged at $\mathrm{N}$-terminal extreme of PTP tag by indirect immunofluorescence microscopy; C: analysis of protein expressed by TcCLB.506825.40 gene; C1: polyacrylamide gel electrophoresis of the purified protein fused to a hexahistidine tag; C2: WB analysis using a specific anti-TcCLB.506825.40 antibody and total extract of WT epimastigotes (epi) and metacyclic trypomastigotes (meta); C3: WB analysis using a specific anti-TcCLB.506825.40 antibody and protein extracts of epi and meta expressing the conserved hypothetical protein (TcCLB.506825.40).The lower band (a) corresponds to the endogenous protein and the upper band (b) corresponds to the GFP-tagged protein expressed in the pTcGFPN-CO 1.1 vector; D: subcellular localisation of TcCLB.506825.40 protein fused with GFP in T. cruzi epi parasites; DIC: differential imaging contrast; K: kinetoplast; N: nucleus. Bar $=5 \mu \mathrm{m}$ (B), $10 \mu \mathrm{m}$ (D).

Another improvement in the 1.1 version consists in the possibility of choosing whether using $\mathrm{N}$ or $\mathrm{C}$-terminal fusion tags. This represents a very important feature since signal peptides located at the N-terminal region of proteins might be hidden when fluorescent tags in this portion are used (Simpson et al. 2000).

In the previous version of the platform, to sequentially purify protein complexes we used a TAP tag, which is composed by a duplicated ProtA followed by Tobacco Etch Virus (TEV) protease site and calmodulin binding peptide. However, the second step of TAP tag purification was not efficient (Batista et al. 2010), issue previously reported in the literature and overcame by using a PTP tag (Shimanski et al. 2005). To offer an alternative to TAP tag, our vectors now have either a PTP or a ProtC tag. The PTP tag allows performing two steps purification whereas $P$ rotC tag on its own can be used for single step protein complex purifications.

The vectors from 1.1 version of $\mathrm{p} T c \mathrm{GW}$ platform are compatible with both high and low-throughput studies and had already been successfully used in different studies (Batista at al. 2013, Inoue at al. 2014, Kalb at al. 2014). Altogether the alterations presented at $\mathrm{p} T c \mathrm{GW} 1.1$ platform were designed to easily allow any further customisation and are available for scientific community.

\section{ACKNOWLEDGEMENTS}

To Arthur Günzl, for providing the $\mathrm{pC}-\mathrm{PTP}-\mathrm{NEO}$ and $\mathrm{pN}$ PURO-PTP plasmids.

\section{REFERENCES}

Batista CM, Kalb LC, Moreira CM, Batista GT, Eger I, Soares MJ 2013. Identification and subcellular localization of $T_{c} \mathrm{HIP}$, a putative Golgi zDHHC palmitoyl transferase of Trypanosoma cruzi. Exp Parasitol 134: 52-60.

Batista M, Marchini FK, Celedon PA, Fragoso SP, Probst CM, Preti H, Ozaki LS, Buck GA, Goldenberg S, Krieger MA 2010. A highthroughput cloning system for reverse genetics in Trypanosoma cruzi. BMC Microbiology 10: 259.

Clayton C, Shapira M 2007. Post-transcriptional regulation of gene expression in trypanosomes and Leishmania. Mol Biochem Parasitol 156: 93-101.

Coughlin BC, Teixeira SM, Kirchhoff LV, Donelson JE 2000. Amastin mRNA abundance in Trypanosoma cruzi is controlled by a 3'-untranslated region position-dependent cis-element and an untranslated region-binding protein. J Biol Chem 275: 12051-12060.

Dostalova A, Käser S, Cristodero M, Schimanski B 2013. The nuclear mRNA export receptor Mex67-Mtr2 of Trypanosoma brucei contains a unique and essential zinc finger motif. Mol Microbiol 88: 728-739.

El-Sayed NM, Myler PJ, Bartholomeu DC, Nilsson D, Aggarwal G, Tran AN, Ghedin E, Worthey EA, Delcher AL, Blandin G, Westenberger SJ, Caler E, Cerqueira GC, Branche C, Haas B, Anupama A, Arner 
E, Aslund L, Attipoe P, Bontempi E, Bringaud F, Burton P, Cadag E, Campbell DA, Carrington M, Crabtree J, Darban H, da Silveira JF, de Jong P, Edwards K, Englund PT, Fazelina G, Feldblyum T, Ferella M, Frasch AC, Gull K, Horn D, Hou L, Huang Y, Kindlund E, Klingbeil M, Kluge S, Koo H, Lacerda D, Levin MJ, Lorenzi H, Louie T, Machado CR, McCulloch R, McKenna A, Mizuno Y, Mottram JC, Nelson S, Ochaya S, Osoegawa K, Pai G, Parsons M, Pentony M, Pettersson U, Pop M, Ramirez JL, Rinta J, Robertson L, Salzberg SL, Sanchez DO, Seyler A, Sharma R, Shetty J, Simpson AJ, Sisk E, Tammi MT, Tarleton R, Teixeira S, Van Aken S, Vogt C, Ward PN, Wickstead B, Wortman J, White O, Fraser CM, Stuart KD, Andersson B 2005. The genome sequence of Trypanosoma cruzi, etiologic agent of Chagas disease. Science 309: 409-415.

Inoue AH, Serpeloni M, Hiraiwa PM, Yamada-Ogatta SF, Muniz JR, Motta MC, Vidal NM, Goldenberg S, Avila AR 2014. Identification of a novel nucleocytoplasmic shuttling RNA helicase of trypanosomes. PloS One 9: e109521.

Kalb LC, Frederico YC, Batista CM, Eger I, Fragoso SP, Soares MJ 2014. Clathrin expression in Trypanosoma cruzi. BMC Cell Biology 15: 23.

Kramer S, Marnef A, Standart N, Carrington M 2012. Inhibition of mRNA maturation in trypanosomes causes the formation of novel foci at the nuclear periphery containing cytoplasmic regulators of mRNA fate. $J$ Cell Sci 125: 2896-2909.
Liang XH, Haritan A, Uliel S, Michaeli S 2003. Trans and cis splicing in trypanosomatids: mechanism, factors and regulation. Eukaryotic Cell 2: 830-840.

Martinez-Calvillo S, Lopez I, Hernandez R 1997. pRIBOTEX expression vector: a pTEX derivative for a rapid selection of Trypanosoma cruzi transfectants. Gene 199: 71-76.

Schimanski B, Nguyen TN, Gunzl A 2005. Highly efficient tandem affinity purification of trypanosome protein complexes based on a novel epitope combination. Eukaryotic Cell 4: 1942-1950.

Simpson JC, Wellenreuther R, Poustka A, Pepperkok R, Wiemann S 2000. Systematic subcellular localization of novel proteins identified by large-scale cDNA sequencing. EMBO Rep 1: 287-292.

Vazquez MP, Levin MJ 1999. Functional analysis of the intergenic regions of $T c$ P2beta gene loci allowed the construction of an improved Trypanosoma cruzi expression vector. Gene 239: 217-225.

Weatherly DB, Boehlke C, Tarleton RL 2009. Chromosome level assembly of the hybrid Trypanosoma cruzi genome. BMC Genomics 10: 255 .

Wen LM, Xu P, Benegal G, Carvaho MR, Butler DR, Buck GA 2001. Trypanosoma cruzi: exogenously regulated gene expression. Exp Parasitol 97: 196-204. 